\title{
Encounter based Routing and Buffer Management Scheme in Delay Tolerant Networks
}

\author{
Paresh C. Patel \\ Department of Information Technology \\ G H Patel College of Engineering and Technology, \\ Vallabh Vidyanagar, Gujarat, India
}

\author{
Nikhil N. Gondaliya \\ Department of Information Technology \\ G H Patel College of Engineering and Technology, \\ Vallabh Vidyanagar, Gujarat, India
}

\begin{abstract}
Delay Tolerant Networks (DTNs) are one of the most interesting evolutions of MANETs where frequent disconnection of networks is there so that end to end path not establish between source to destination. When seeing routing protocols of DTNs, a forwarding and buffer management are important to be considered for successful message delivery. 'Encounter Based Routing (EBR)' which increase delivery ratio by decreasing overhead and delay using the encounter value of the nodes. EBR takes the average Encounter Value (EV) of the node and decide that how many copies of the message to be sent to the other nodes. Higher the EV value, the node will get more number of message copies. We propose the modified EBR which optimizes the performance of the existing EBR. We consider the change of rate of EV with respect to time and then intelligently decide the number of replicas to be sent to the encountered nodes. The modified EBR scheme performs better in terms of delivery ratio than the existing EBR protocol. We also propose the buffer management policy using EV of the node to decide the dropping of the message in the Epidemic routing protocol. These schemes are HEV (High Encounter Value) and LEV (Low Encounter Value) and they are compared with the existing traditional policies such as LIFO and FIFO. The simulation results show that our proposed dropping policy improves the overhead ratio, delivery delay and hop count metrics than the existing ones.
\end{abstract}

\section{General Terms}

Routing scheme, Performance, Buffer management policy.

\section{Keywords}

DTNs, Epidemic, Spray and Wait (SnW), Encounter Routing Protocol (EBR), ONE Simulator, LIFO, FIFO, EHV (Encounter High Value) and ELV (Encounter Low Value).

\section{INTRODUCTION}

Delay Tolerant Networks is the wireless network where endto-end path from a source node to destination node is not fix because it has frequent disconnection of networks are there. When two node contact with each other than communication opportunity establish. It that time node can forward the message to other node. If any node has a message to send but it is not communicate with another node or contact with other node. It store the message the data until there is communication establish with other node. Communicate with any two node each other in such a networks it called contact [3].

Delay Tolerant Networks (DTNs) use the store-carry-forward method for transport the data where no infrastructure happen. Also an end-to-end path not available from one node to another node. Routing protocols take benefit of temporal paths made in such a network as nodes encounter each other and exchange data. But it has no guarantees to end-to-end path available in networks. Many routing protocols apply techniques for reduce the delay, network resources in limited bandwidth and the possible to increase end-to-end delay [1].

EBR usages the encounter value of the node. Number of time one particular node contact with another nodes that count in each nodes store record of that buffer. Using that record of each node the encounter value. Node which have higher EV value should send the replica of message so that the chance of delivered message is higher and no more number of message copies in the networks so that overhead ratio also lower as related to other protocols. It usages the present rate of encounters and averages [3]. Here in this paper modified EBR protocol to consider the variation in rate of encounters over time, so that particular time how many encounter values of nodes and change that value over the time. Based on that value node can forward the number of message copies to the encounter nodes.

Buffer management is very important in DTN because the combination of long-term storage of messages and the message replication places a high bandwidth and storage overhead on nodes. When the buffer is full, often in order to accommodate a new message a DTN node will have to drop an important message [9]. If an efficient dropping policy is implemented that can prioritize the message drop sequence. We proposed the efficient dropping policy HEV and LEV that can be drop based on the high and low EV message passed the nodes which compare with the traditional policy LIFO and FIFO.

This paper is prepared as follows. In section 1, Introduction about DTNs. Section 2, a brief description of related work and our contribution of protocols. Section 3, Description of proposed modified encounter based routing protocol and buffer management policy and techniques. Also simulation setup and results are discussed. We achieve the paper in section 4 with a discussion of future work.

\section{RELATED WORKS}

In DTNs, The main challenge for routing is the dynamic topology due to intermittent connectivity and lack of an endto-end path between two nodes. It contain static and dynamic nodes like mobiles. The connections between two static nodes could be up or down. But dynamic nodes are difficult because network topology changes as nodes move. In such a condition the connection between pairs of nodes is brought about when they come into range of each other. So that, mobility effects intermittent connectivity [2]. The following part discusses the existing routing protocols in brief. 


\subsection{Epidemic Routing Protocol}

Epidemic routing protocol provide data delivery in disconnected networks because there is no certain present route for connecting pairs. Epidemic routing is to distribute replicas messages to hosts within connected nodes of ad hoc networks. This way, messages are speedily spread over connected nodes of the networks. The overall goal of epidemic routing is to maximize message delivery ratio and minimize message delivery latency, while also reduce the joint system assets spent in message delivery [7].

Epidemic protocol replicates message copies into the network for improving the probability of message delivery. On the other hand, spray and wait protocol spread the limited copies of the messages into the network and it also provides the better delivery and low overhead in the networks.

\subsection{Spray and Wait (SnW) Routing Protocol}

$\mathrm{SnW}$ routing protocol that tries to gain higher delivery ratio and low overhead ratio in replica based routing also it use low resources of forwarding-based routing. SnW protocol has two phases: "Spray phase" and "Wait phase." For the duration of the spray phase, the source of the message is responsible for spray or delivery one replica to destination node. When a node receives the replica, it go in the wait phase, after direct transmit to the destination node [5].

SnW has a two versions, both version are identify based on the how many number of copies reach to the distinct nodes during the spray phase of SnW. First version is for the source to spread a single copy of the message to the first distinct nodes it meets after the message is created. A second version Binary SnW. the source starts spread the message copies. If any first node which has no any copy encounters. Both nodes, then transfer half of the total number of copies they have to future nodes they meet that have number of message copies. When a node have only one copy then it shifts into the wait phase where it waits for a direct transmission chance with the destination node [5].

The advantage of Binary SnW is that messages are spread faster than the source. SnW combined the profits both the simplicity of Epidemic and the consistency of the direct transmission [6].

\subsection{Encounter-Based Routing (EBR) Protocol}

Encounter Based Routing (EBR) that provides high delivery ratios similar to flooding-based protocols, while keeping low overhead ratio. EBR use encounter value for forward the copies of message based on the encounter value future rate of node can be predicted by the encounter rate of past data. It is quota based routing so that limited number of message copies in the networks so that it usages limited resource of networks [4].

In EBR, it take the node's encounter rate which is purely local metric, using small no of variable it can be tracked. Each node is responsible for keeping their past rate of encounter average. When two nodes encounter, the comparative ratio of their own rates of encounter regulates the suitable segment of message replicas the nodes should interchange [4].

\subsection{Buffer Management Techniques}

Buffer storage management is an important which must be managed in efficient way. It includes two different tasks:
An Encounter Value (EV) "the node's past rate of encounters as an exponentially weighted moving average" and Current Window Counter (CWC) "It is used to obtain information about the number of encounters in the current time interval."

EBR considers the current rate of encounters based on this rate of encounter it forward the no of copies to the encounter nodes.

\section{PROPOSED ROUTING SCHEME}

In this section, Description about modified EBR Routing protocol with proposed algorithm and some buffer management techniques like FIFO, LIFO, HEV and LEV. Also simulation results discuss.

\subsection{Modified EBR Routing Protocols}

This routing protocol provides the same functionality, but it gives the better result compared to the existing EBR routing protocol. To track a node's rate of encounter, every node maintains two pieces of local information: an encounter value $(\mathrm{EV})$, and a current window counter (CWC). EV represents the node's past rate of encounters and CWC is used to obtain information about the number of encounters in the current time interval. EV is updated to account for the most recent CWC in which rate of encounter information was obtained with respect to time [4]. Updates to $\mathrm{EV}$ are computed as follows using equation 1 .

$$
E V \leftarrow \alpha \cdot \frac{d C W C}{d t}+(1-\alpha) \cdot \frac{d E V}{d t}
$$

This exponentially weighted moving average places an emphasis proportional to $\alpha$ on the most recent complete CWC with respect to time. How many message send one node to another node that can be decided by the EV represents a prediction of the future rate of encounters for each node per time interval, the node with the highest EV represents a higher probability of successful message delivery. So that, when two nodes meet, they compare their EVs. The number of replicas of a message transferred during a contact opportunity is proportional to the ratio of the EVs of the nodes. For two nodes $\mathrm{A}$ and $\mathrm{B}$, for message $\mathrm{M}_{\mathrm{i}}$., node $\mathrm{A}$ sends replicas of Mi., where $M_{i}$ is the total number of $M_{i}$ Replicas stored at node A [4].

$$
\begin{aligned}
& M_{\text {send }} \leftarrow\left[M_{i} * \frac{E V_{B}}{E V_{A}+E V_{B}}\right] \\
& \text { 3.1.1 Algorithm:-1 Modified EBR Routing }
\end{aligned}
$$

$$
\begin{aligned}
& \text { 1. } \text { If time } \geq \text { nextUpdate then } \\
& \text { 2. } \\
& \text { 2. } \leftarrow \alpha \cdot \frac{d C W C}{d t}+(1-\alpha) \cdot \frac{d E V}{d t} \\
& \text { 3. } \mathrm{CWC} \leftarrow 0 \\
& \text { 4. } \text { nextUpdate } \leftarrow \text { time }+\mathrm{Wi} \\
& \text { 5. } \text { end if } \\
& \text { 6. If Contact } \mathrm{C} \text { available then } \\
& \text { 7. } \text { for All messages Mi in local buffer do } \\
& \text { 8. } \mathrm{Mi} \leftarrow \text { Mi.numOfReplicas } \\
& \text { 9. } \\
& M_{\text {send }} \leftarrow\left[M_{i} * \frac{E V_{B}}{E V_{A}+E V_{B}}\right] \\
& \text { 10. Send Msend replicas of Mi to node C } \\
& \text { 11. } \text { end for } \\
& \text { 12. } \text { end if }
\end{aligned}
$$

message scheduling and dropping policy [9]. As the contact period between the node pair is not predictable so, node has to decide that which message are transferred first is called 
message scheduling. When buffer runs out of space then node has to decide that which message is dropped first is called dropping policy. Here we do concern with message dropping policies. Certain buffer management techniques are as follows:

\section{First In First Out (FIFO)}

This technique drops the messages on the basis of the order in which they arrived in the buffer, for example the first message that arrived the queue will be the first to be dropped [9].

\section{Last In First Out (LIFO)}

This method drops the messages on the basis of the order in which they entered into the buffer, for example the last message that arrived the queue will be the first to be released [9].

\section{High Encounter Value (HEV)}

This technique drops the messages on the basis of the encounter value of that node in which they entered into the buffer, for example the message passed away higher EV nodes that message entered the queue will be the first to be dropped.

\section{Low Encounter Value (LEV)}

This technique drops the messages on the basis of the EV of that node in which they entered into the buffer, for example the message passed away lower EV nodes that message entered the queue will be the first to be dropped.

\subsection{SIMULATIONS \& RESULTS}

In this section discusses the performance metrics used to evaluate the routing protocols and simulation results.

\subsubsection{The Performance Metrics}

We choose the following performance metrics [3] which are mostly used in the literature for evaluating the routing protocol:

I. Delivery ratio. It is defined as the proportion of the number of messages successfully delivered to the destination of the total number of messages created [3].

II. Overhead ratio, which is defined as the ratio of total number of relayed messages and delivered message different to the total number of messages delivered [3].

III. Average latency. It is defined as the average time need to deliver a message to the destination nodes [3].

IV. Average hop count. It is average number of hops that message require to reach the end node [3].

V. Efficiency Measurement which can be defined as ratio of the delivery ratio to overhead ratio than can provide the routing efficiency of epidemic routing protocol [9].

\subsubsection{Experimental Data Sets}

Choose following two datasets from two different environment such as campus and conference to verify the effectiveness of the routing protocols.

Cambridge: This trace includes Bluetooth sightings by groups of users carrying small devices around the city of Cambridge, UK. Device users in experiment mostly contained of students from Cambridge University at the reception of the Computer Lab, in which maximum number of the experiment applicants are students [11].

Infocom06: This is Trace of Bluetooth sightings by groups of users carrying small devices (iMotes) for four days. It carries a portable Bluetooth transmitter device. This file contains 98 nodes and require the 987529 second. This trace data contains groups of users carrying small Bluetooth devices for 4 days in Conference IEEE Infocom [11].
Table 1. Simulation Parameter for Cambridge and Infocom06 Data Set

\begin{tabular}{|l|l|l|}
\hline PARAMETERS & \multicolumn{2}{|l|}{ VALUE } \\
\hline Experimental Dataset & Cambridge & Infocom06 \\
\hline Simulation Time & $987529 \mathrm{sec}$ & $342915 \mathrm{sec}$ \\
\hline Number of Nodes & 36 & 98 \\
\hline Interface & Bluetooth Interface \\
\hline Transmit Speed & 250k(2 Mbps) \\
\hline Message copies & 4 \\
\hline Buffer Size & 5 To 50 M \\
\hline Routing Protocols & $\begin{array}{l}\text { Epidemic, SaW, EBR and } \\
\text { Modified EBR }\end{array}$ \\
\hline Message Size & 200k,400k,600k,800k,1M \\
\hline Message Interval & 5 to 40 sec \\
\hline Message TTL & 10m to 4Day \\
\hline
\end{tabular}

\subsection{Result Analysis of Routing Protocols for Cambridge Data Set}

In this section, Analysis result of routing protocol approach and buffer management approach with epidemic, SnW, EBR and modified EBR routing protocol using the evaluation parameter overhead ratio, delivery ratio, latency avg. and hop count avg. for routing approach and HEV, LEV, LIFO and FIFO for buffer management.

\subsubsection{Impact of varying Buffer Size}

Figure 1. Shows the varying of buffer size on delivery ratio, overhead ratio, latency average and hop count average for Epidemic, SnW, EBR and Modified Encounter Based Routing protocols. Takes constant parameters are message interval $(25-35 \mathrm{sec})$, message size $(250 \mathrm{k}-1 \mathrm{M})$ and TTL (300 min.) on Cambridge dataset. From the graph it can be observed that in higher values of buffer sizes of modified EBR provide up to $7-8 \%$ of higher delivery ratio as compared to epidemic, $\mathrm{SnW}$

and EBR routing protocols. In small values of buffer size modified EBR provide up to 2-3\% lower latency as compare to the other routing protocols

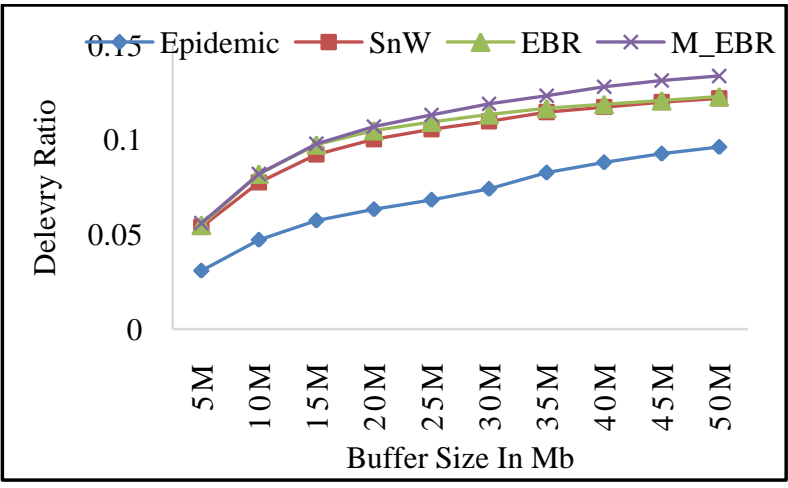

(a) 


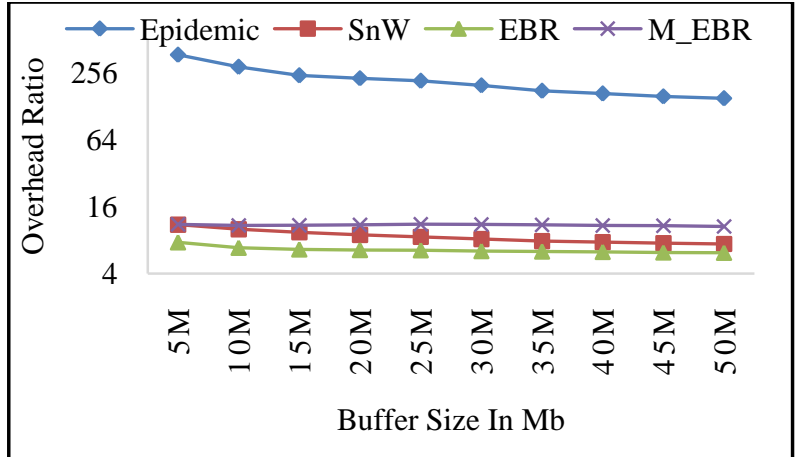

(b)

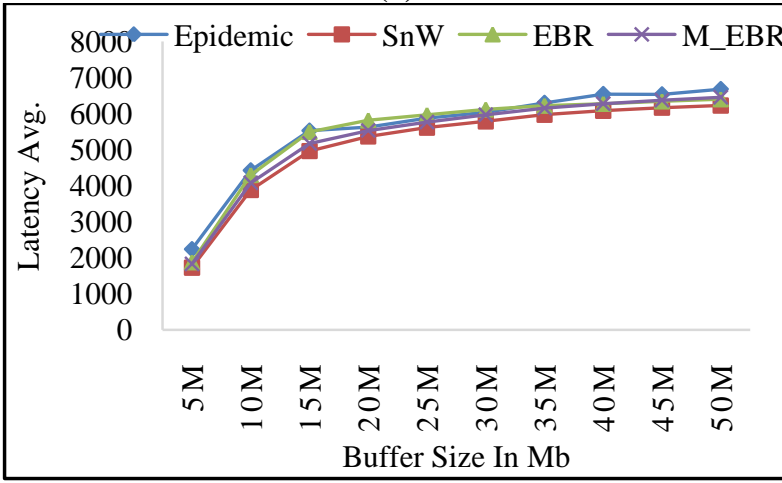

(c)

Figure 1. Impact of Varying Buffer Size with (a) Delivery Ratio (b) Overhead Ratio (c) Latency Avg. (d) Hop Count Avg.

\subsubsection{Impact of varying Message TTL}

Figure 2 Shows the varying of message TTL on delivery ratio, overhead ratio, latency average and hop count avg. for epidemic routing, SnW, EBR and modified EBR protocols. Take the constant values are buffer size $(35 \mathrm{M})$, message size (250k-1M) and message interval (25-35 Sec.) on cambridge

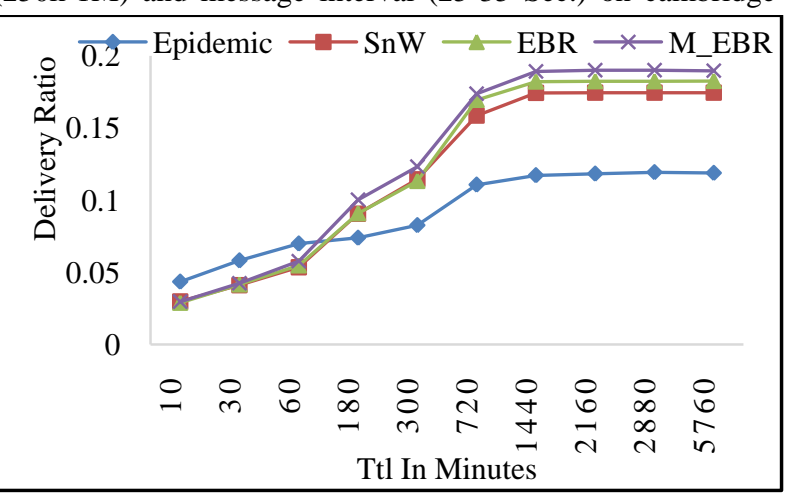

(a)

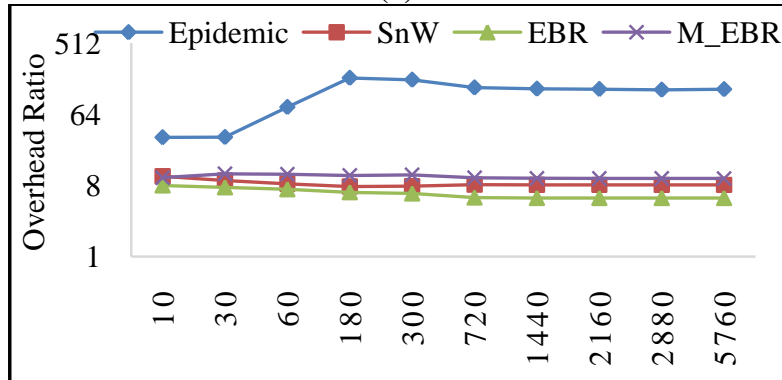

Ttl In Minutes

(b)

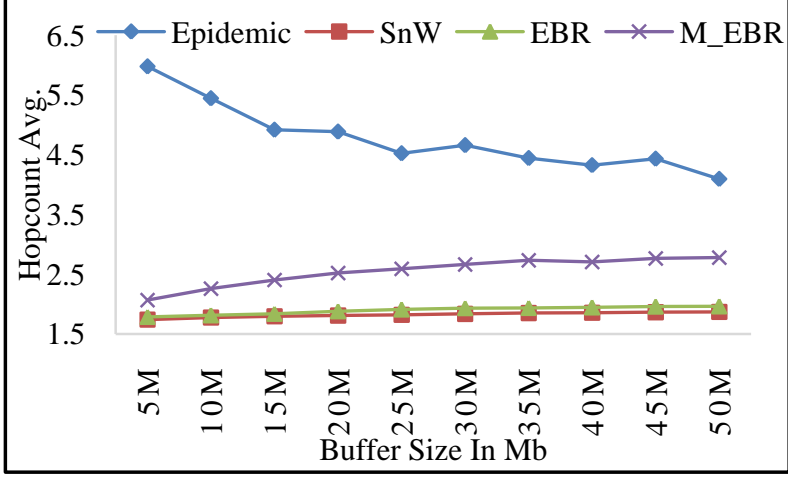

(d) dataset. From the graph it can be observed that in TTL of 60300 minutes of modified EBR provide up to 4-9 \% of higher delivery ratio and same TTL values of modified EBR provide up to 3-6\% lower latency as compare to epidemic, SnW and EBR routing protocols. After greater values of modified EBR (720 Min.) provide constant in higher TTL values.

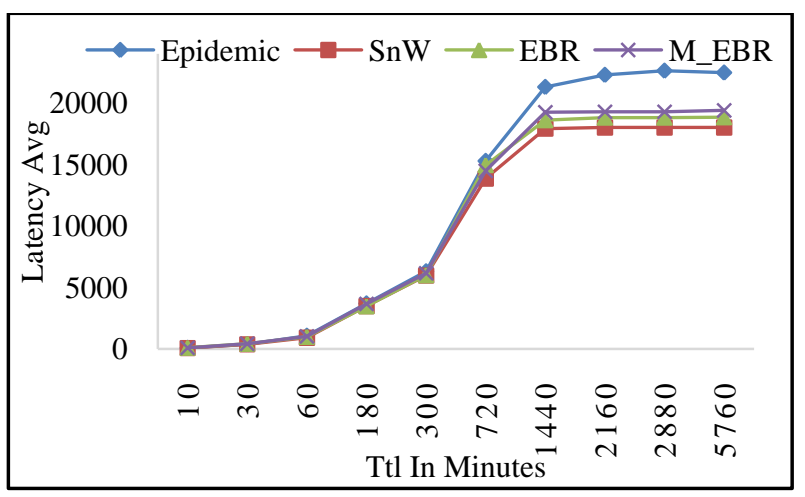

(c)

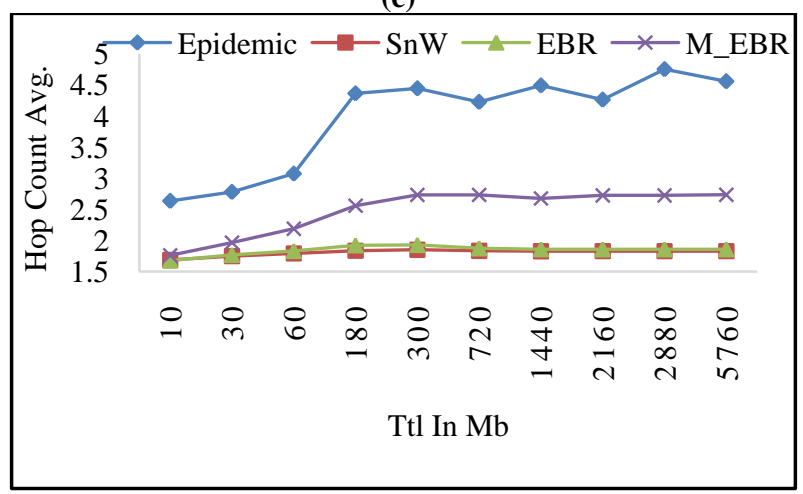

(d)

Figure 2. Impact of Varying TTL with (a) Delivery Ratio (b) Overhead Ratio (c) Latency Avg. (d) Hop Count Avg. 


\subsubsection{Impact of varying Message Size}

Figure 3 Shows the varying of message size on delivery ratio, overhead ratio, latency average and hop count avg. for epidemic routing, SnW, EBR and modified EBR protocols. Take constant parameter in this simulation are buffer size (35M), message TTL (300 Min.) and message interval (25-35

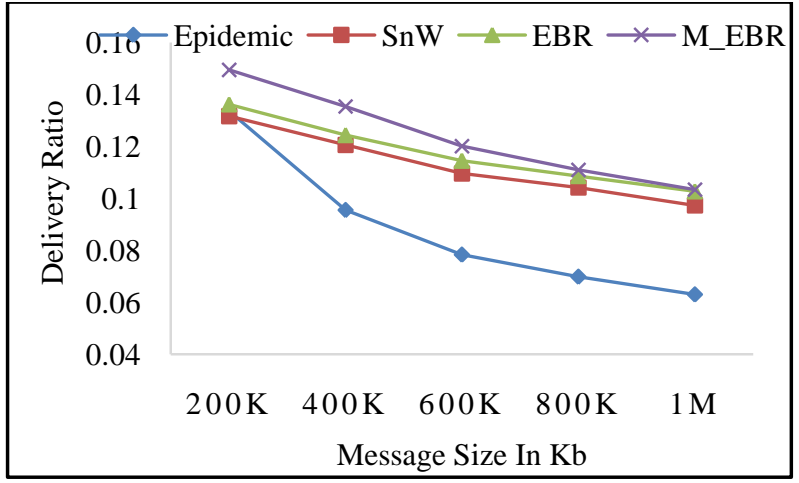

(a)

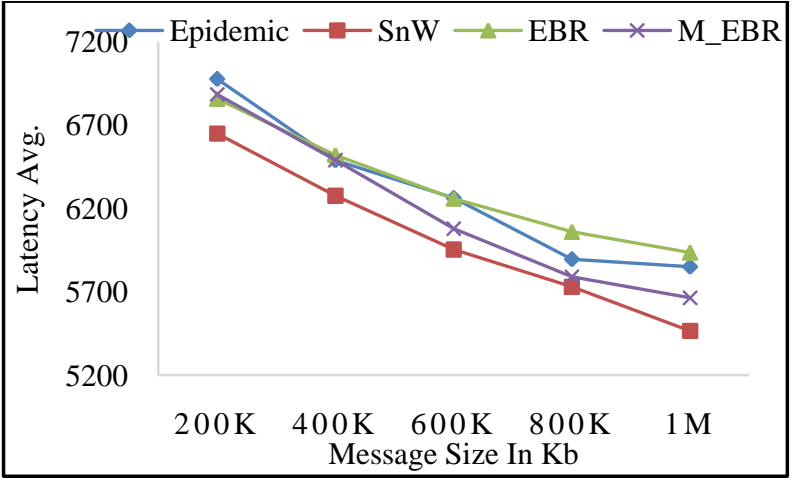

(c) sec.) on cambridge dataset. From the graph it can be noticed that in message size $(200-600 \mathrm{~kb})$ of modified EBR provide up to $5-9 \%$ of the higher delivery ratio and message size of $600 \mathrm{k}-1 \mathrm{M}$ values of modified EBR provide up to 3-5 \% lower latency as compare to epidemic, SnW and EBR routing protocols.

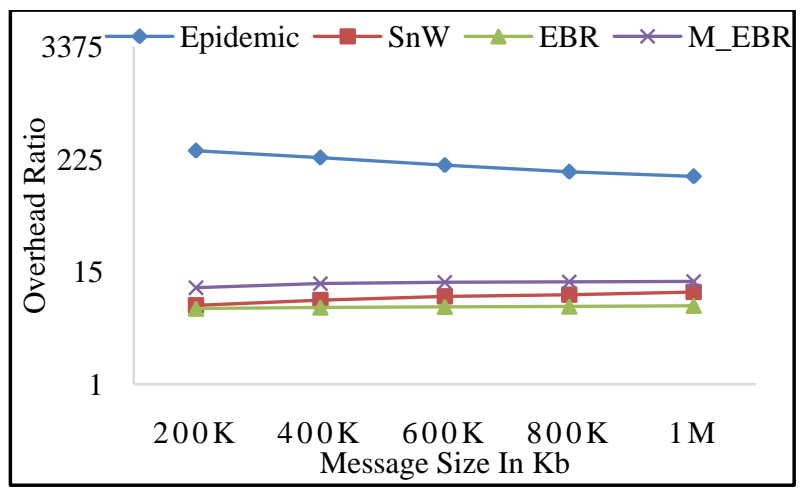

(b)

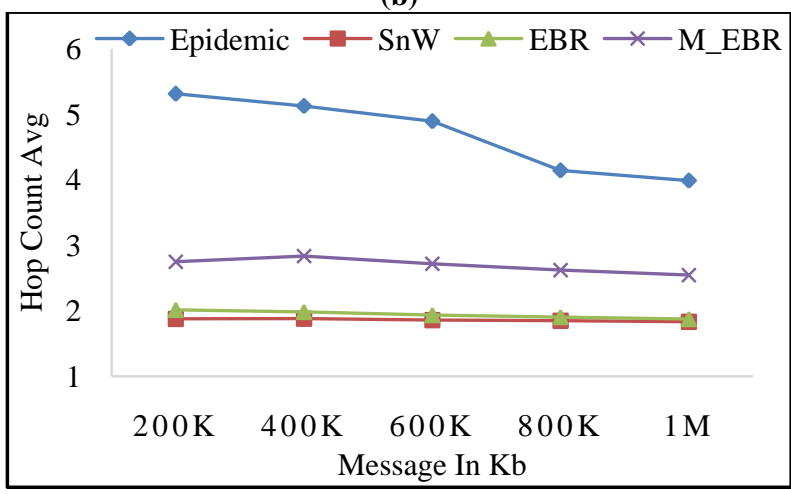

(d)

Figure 3. Impact of Varying Message Size in Kb with (a) Delivery Ratio (b) Overhead Ratio (c) Latency Avg. (d) Hop count avg.

\subsubsection{Impact of varying Message Interval}

Figure 4 Shows the varying of message interval on delivery ratio, overhead ratio, latency average and hop count average for epidemic routing, SnW, EBR and modified EBR protocols. Take constant values are buffer size $(35 \mathrm{M})$, message size (250k-1M) and TTL (300 Min.) on cambridge

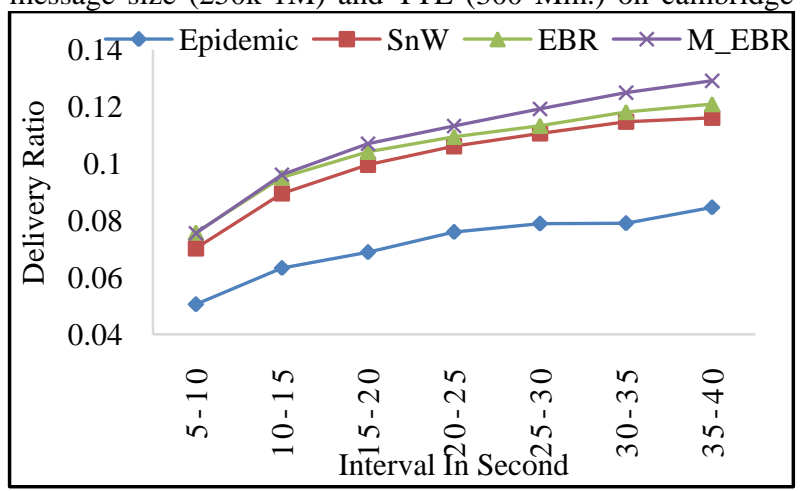

(a) dataset. From the graph it can be noticed that in higher values of message interval of modified EBR provide up to 3-6\% of higher delivery ratio and small values of message interval of modified EBR provide up to 2-5\% lower latency as compared to epidemic, SnW and EBR protocols.

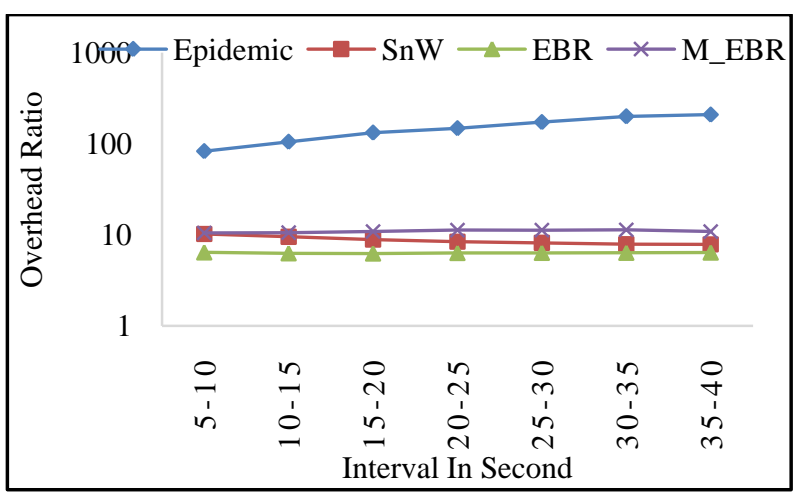

(b) 


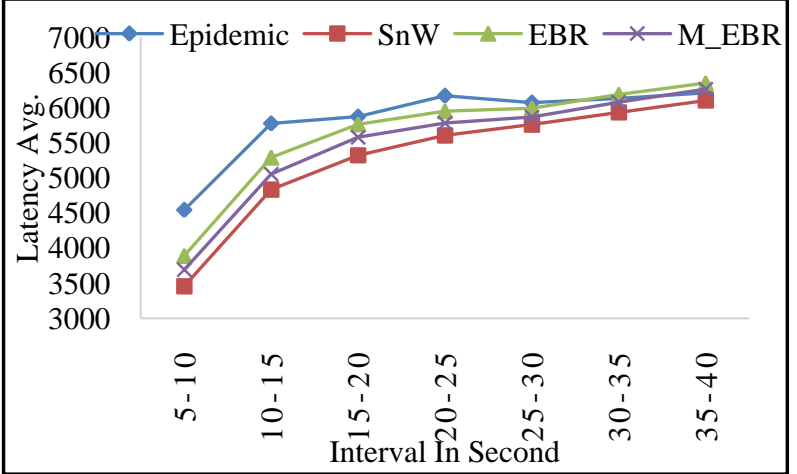

(c)

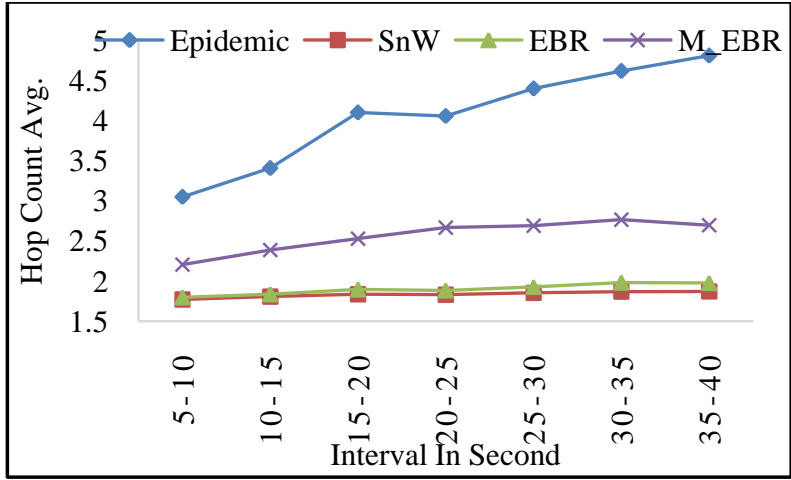

(d)

Figure 4. Impact of Varying Message Interval in second with (a) Delivery Ratio (b) Overhead Ratio (c) Latency Avg. (d) Hop count avg.

\subsection{Result Analysis of Buffer Management \\ Policies for Cambridge Data Set}

3.5.1 Impact of varying Efficiency on Buffer size Our routing approaches HEV and LEV give the higher values compare the traditional routing protocol buffer management policies LIFO and FIFO. The overall routing efficiency of delivery and overhead ratios in various buffer sizes that give the better result as compare to the traditional approach shown in figure 5 .

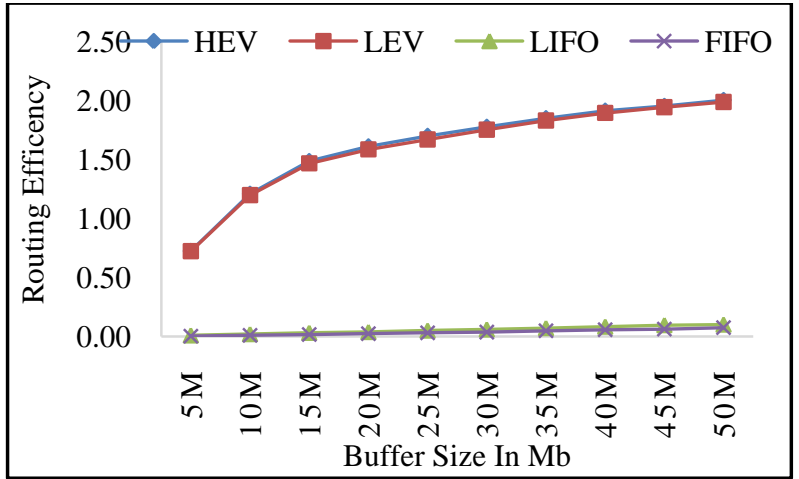

Figure 5. Routing Efficiency on Buffer Size

\subsubsection{Impact of varying Efficiency on Message} $T T L$

It initially in small TTL values our approaches HEV and LEV provide better efficiency than traditional approaches. We can show in figure 6 that HEV and LEV give better Efficiency than LIFO and FIFO.

\subsubsection{Impact of varying Efficiency on Message} Size

In different message size the routing efficiency of HEV and LEV is higher than tradition strategies LIFO and FIFO shown on figure 7.

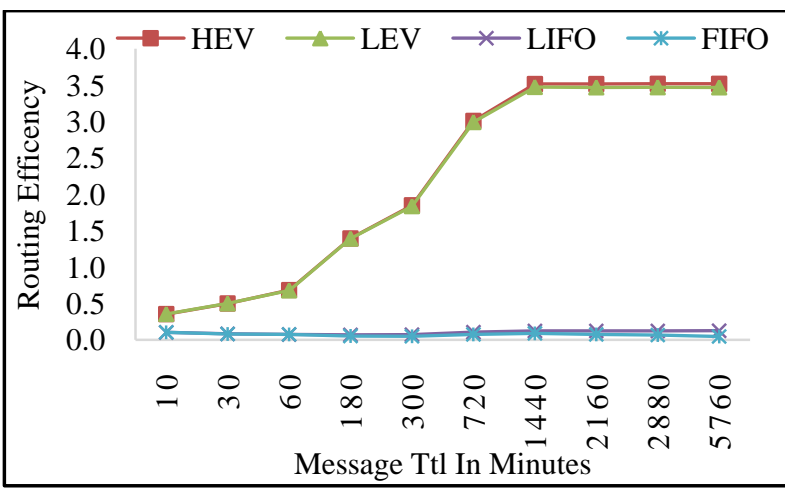

Figure 6. Routing Efficiency on Message TTL

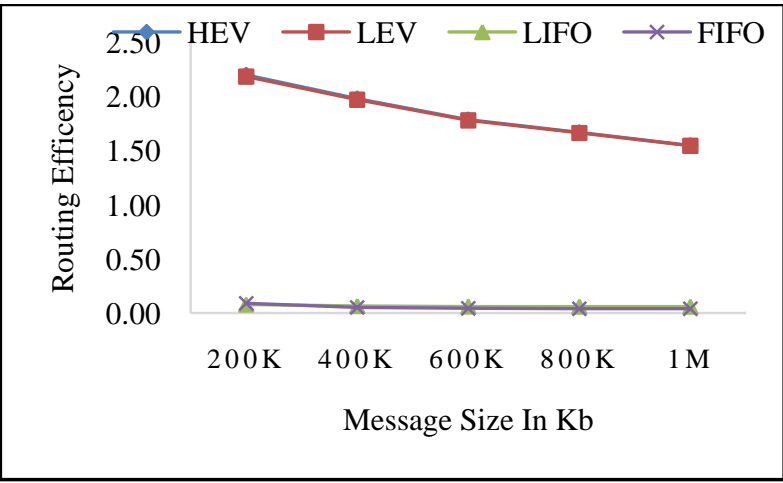

Figure 7. Routing Efficiency on Message Size 


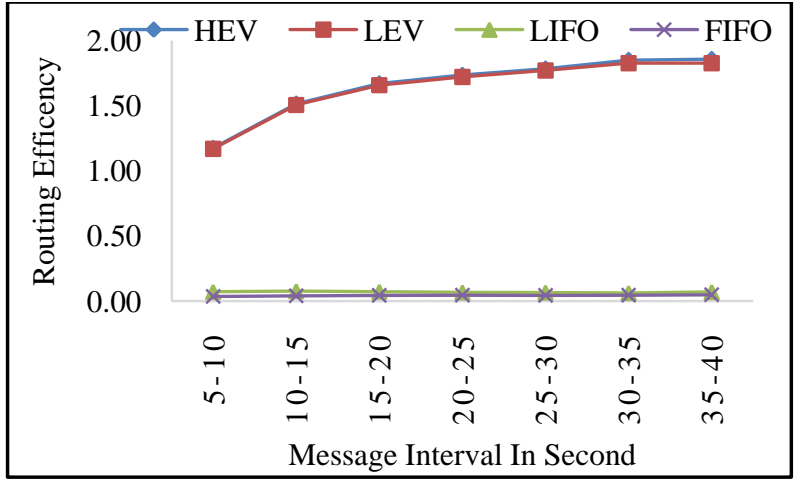

Figure 8. Routing Efficiency on Message Interval

\section{CONCLUSION AND FUTURE WORK}

Based on the simulation result, the modified EBR gives 7-8 \% better delivery ratio compared to the EBR protocol for the higher values of buffer size, interval, message TTL and message size. Average latency of modified EBR is lower up to 3-6 \% lower than the original EBR protocol. Same simulation result provide on the infocom06 real trace data set.

We have also proposed the buffer management schemes (HEV and LEV) which select the message to drop based on the Encounter Value associated with the message. These schemes performs better than traditional policies such as LIFO and FIFO in terms of overhead ratio, delivery delay and hop count for the Epidemic routing protocol.

We have evaluated the performance of modified EBR on a single dataset of campus and conference environments. In future work, it can be applied to a number of datasets to verity its correctness. The proposed buffer management scheme can also be compared to other policies such as most forwarded first (MOFO), drop random, drop least recently received and based on the message size.

\section{ACKNOWLEDGMENTS}

We are extremely thankful to entire Information Technology Department, faculty and staff, for helping us in every conceivable way during my course of study in this work.

\section{REFERENCES}

[1] SuvarnaPatil, Geetha R. Chillerge "Delay Tolerant Networks - Survey Paper" ISSN: 2248-9622, Vol. 4, Issue 2(Version 2), pp.21-25 February 2014, IJERA.
[2] Yue Cao and Zhili Sun,"Routing in Delay/Disruption Tolerant Networks: A Taxonomy, Survey and Challenges" Communications Surveys \& Tutorials, Vol.15, No. 2, Second Quarter 1553-877X/13/\$31.00@ 2013 IEEE.

[3] Salman Ali, Junaid Qadir, Adeel Baig, "Routing Protocols in Delay Tolerant Networks - A Survey" ICET 978-1-4244-8058-6/10/\$26.00 @2010 IEEE.

[4] Samuel C. Nelson, Mehedi Bakht, and Robin Kravets "Encounter-Based Routing in DTNs" INFOCOM 978-14244-3513-5/09/\$25.00 @2009 IEEE.

[5] Thrasyvoulos Spyropoulos, Konstantinos Psounis, Cauligi S. Raghavendra "Spray and Wait: An Efficient Routing Scheme for Intermittently Connected Mobile Networks" SIGCOMM'05 Workshops, ACM 1-59593026-4/05/0008.August 22-26, @ 2005.

[6] Eung-Hyup Kim, Jae-Choong Nam, Jae-In Choi, You-Ze Cho "Probability-based Spray and Wait Protocol in Delay Tolerant Networks" ICOIN 978-1-4799-36892/14/\$31.00 @2014 IEEE.

[7] Amin Vahdat and David Becker "Epidemic routing for partially connected ad hoc networks" Technical Report CS-200006, Duke University, April 2000.

[8] Ker Anen, "Opportunistic Network Environment Simulator. Special assignment report, helsinki University of Technology," Department of Communications and Networking, May 2008.

[9] Sweta Jain, Meenu Chawla, "Survey of buffer management policies for delay tolerant networks", doi: 10.1049/joe.2014.0067 The Journal of Engineering.

[10] Anita Rani, Sangeeta Rani, Harminder Singh Bindra. "Performance Evaluation of MaxProp Routing Protocol with DL, FIFO, DLA and MOFO Buffer Management Techniques in DTN under Variable Message Buffer Size" IJERT, ISSN: 2278-0181 Vol. 3 Issue 2, February -2014 .

[11] CRAWDAD Community for Wireless Data At Dartmouth,http://crawdad.org/keyword-DTN.htm 